\title{
Partner of Sld five 3: a potential prognostic biomarker for colorectal cancer
}

\author{
Xiaoli Sun ${ }^{1}$, Wu Sui ${ }^{2}$, Miaoling Huang ${ }^{3}$, Yeli Wang ${ }^{3}$, Yuanjie Xuan ${ }^{3}$ and Zaiqiu Wang ${ }^{3 *}$
}

\begin{abstract}
Background: Partner of Sld five 3 (PSF3) is a member of the evolutionarily conserved heterotetrameric complex "Go-Ichi-Ni-San" (GINS), which consists of SLD5, PSF1, PSF2, and PSF3. Previous studies have suggested that some GINS complex members are upregulated in cancer, but the status of PSF3 expression in colorectal cancer has not been investigated.
\end{abstract}

Methods: We investigated the status of PSF3 expression in 137 consecutive resected colorectal caners by quantitative reverse-transcription polymerase chain reaction. Univariable and multivariable Cox regression analyses were performed to assess independent prognostic factors for overall survival in colorectal cancer.

Results: In 137 restected colorectal cancer samples, median messenger RNA (mRNA) expression levels of PSF3 were significantly higher in tumor tissues $\left(1.35 \times 10^{-3}\right.$, range $2.88 \times 10^{-4}$ to $\left.3.16 \times 10^{-2}\right)$ than in adjacent normal tissues $\left(2.94 \times 10^{-4}\right.$, range $5.48 \times 10^{-5}$ to $\left.1.27 \times 10^{-3}\right)(P<0.05)$. Moreover, high expression of PSF3 in tumor tissues was associated with shorter disease-free survival and overall survival. When analyzed with a Cox regression model, the PSF3 expression was an independent prognostic factor for overall survival. In addition, in patients with early stage (stage I and II) colorectal cancer, the overall survival rate of the high PSF3 expression group was significantly lower than that of the low PSF3 expression group $(P<0.001)$.

Conclusions: The PSF3 expression plays an important role in the progression of colorectal cancer and acts as a factor significantly affecting the prognosis of patients.

Virtual Slides: The virtual slide(s) for this article can be found here: http://www.diagnosticpathology.diagnomx.eu/ vs/13000_2014_217

Keywords: Partner of Sld five 3, Colorectal cancer, Overall survival

\section{Background}

Partner of Sld five 3 (PSF3) is a member of the highly evolutionarily conserved tetrameric complex termed GoIchi-Ni-San (GINS), composed of SLD5, PSF1, PSF2, and PSF3. In yeast, the GINS complex associates with the Minichromosome maintenance (MCM) 2-7 complex and CDC45, and this "CMG complex" (CDC45/MCM2$7 /$ GINS) regulates both the initiation and progression of DNA replication [1-3]. Thus, it has been suggested that GINS is involved in DNA replication in Xenopus and human [4-6]. However, a recent study suggested that PSF1/PSF2 is associated with the response to replication

\footnotetext{
* Correspondence: zaiqiu_wang@yeah.net

${ }^{3}$ Anorectal Surgery, Yuhuangding Hospital, No. 20 Yuhuangding East Road, Yantai 264000, Shandong, China

Full list of author information is available at the end of the article
}

stress and acquisition of DNA damage in untransformed human dermal fibroblasts [7]. As it has been reported that DNA replication-associated protein in yeast has diverse functions in different cells, e.g. origin recognition protein Orc1 has a role in determining centrosome copy number [8], the exact functions of GINS components in mammalian cells are not yet clear.

Several recent reports have suggested that PSF1 is required for the acute proliferation of cells, particularly immature cells such as stem cells and progenitor cells and that this protein is useful in the successful detection of cancer stem cells [9-12]. Moreover, previous studies have suggested that some GINS complex members are upregulated in cancer, and some GINS components may be useful in the detection of cancer stem cells $[13,14]$. 
Although several studies have suggested that GINS components play a role in cancer $[15,16]$, the expression status of these components in patients with colorectal cancer has not yet been examined. Therefore, we sought to evaluate the mRNA expression status of PSF3 in surgically resected samples of colorectal cancer tissue. We also investigated whether PSF3 expression in tumor tissues influenced the prognosis of these patients.

\section{Methods}

\section{Patients}

The study population comprised 137 consecutive patients (79 males and 58 females) who were examined and treated at Yuhuangding Hospital between January 2008 and December 2012 for colorectal cancer. All cases underwent complete resection in this study. Details of the clinical and demographic information, prognostic factors, and disease progression were collected prospectively. Of the 137 patients, 46, 54, 22, and 15 had stage I, II, III, and IV tumors, respectively. Forty-two patients were administered postoperiative adjuvant chemotherapy every three weeks for six months (Oxaliplatin $130 \mathrm{mg}$ / $\mathrm{m}^{2} \mathrm{~d} 1+$ Capecitabine $\left.1000 \mathrm{mg} / \mathrm{m}^{2} \mathrm{~d} 1-\mathrm{d} 14\right)$. The study protocol was approved by the institutional review board of Yuhuangding Hospital and the study was conducted according to the principles of the Declaration of Helsinki. All patients provided written informed consent.

\section{RNA isolation and qRT-PCR}

Quantitative reverse-transcription polymerase chain reaction (qRT-PCR) was used to determine the PSF3 expression level. Briefly, total RNA was extracted with Trizol reagent (Invitrogen, Grand Island, NY, USA) and dissolved in water according to the manufacturer's instructions.

Table 1 Association between mRNA expression of PSF3 and clinicopathological characteristics in 137 patients with colorectal cancer

\begin{tabular}{|c|c|c|c|c|}
\hline \multirow[t]{2}{*}{ Variables } & \multirow[t]{2}{*}{ Total } & \multicolumn{2}{|l|}{ PSF3 expression } & \multirow[t]{2}{*}{$P$-value } \\
\hline & & Low level & High level & \\
\hline No. of patients & 137 & 66 & 71 & \\
\hline Age in years, mean \pm SD (range) & $61.2 \pm 7.6(37-86)$ & $59.4 \pm 7.1(37-81)$ & $63.5 \pm 8.8(42-86)$ & 0.634 \\
\hline Gender, M/F & $89 / 48$ & $42 / 24$ & $47 / 24$ & 0.728 \\
\hline Tumor size & & & & $0.013^{*}$ \\
\hline$<5 \mathrm{~cm}$ & 105 & 55 & 50 & \\
\hline$\geq 5 \mathrm{~cm}$ & 32 & 11 & 21 & \\
\hline Depth of wall invasion & & & & $0.006^{*}$ \\
\hline Tis-T2 & 81 & 43 & 38 & \\
\hline T3-T4 & 56 & 23 & 33 & \\
\hline Lymph node metastasis & & & & $0.047^{*}$ \\
\hline Negative & 76 & 39 & 37 & \\
\hline Positive & 61 & 27 & 34 & \\
\hline Distant metastasis & & & & 0.125 \\
\hline No & 118 & 58 & 60 & \\
\hline Yes & 19 & 8 & 11 & \\
\hline TNM stage & & & & $0.008^{*}$ \\
\hline |/II/III/IV & $46 / 54 / 22 / 15$ & $28 / 26 / 8 / 4$ & 18/28/14/11 & \\
\hline Differentiation & & & & $0.021^{*}$ \\
\hline Well/Moderate & 94 & 52 & 42 & \\
\hline Poor & 43 & 14 & 29 & \\
\hline Adjuvant chemotherapy & & & & 0.079 \\
\hline Yes & 42 & 22 & 20 & \\
\hline No & 95 & 44 & 51 & \\
\hline Five-year survival & & & & $0.002^{*}$ \\
\hline Yes & 58 & 35 & 23 & \\
\hline No & 79 & 31 & 48 & \\
\hline
\end{tabular}

M/F Male/Female, PSF3 partner of Sld five 3, SD standard deviation. * Significant $P$-value. 
Relative complementary DNA (cDNA) quantitation for PSF3 and an internal reference gene ( $\beta$-actin) was done using a fluorescence-based, real-time detection method. The sequences of the primer used were as follows: PSF3 forward 5'-TGACAGTCCCGAGAATGCAGA-3' and reverse 5 '-TGCCTACCAGGGCTGAAGTG-3'; $\beta$-actin (internal reference gene) forward $5^{\prime}$-TGGCACCCAGCAC AATGAA-3', reverse 5' -CTAAGTCATAGTCCGCCTA GAAGCA-3'. The PCR mixture consisted $1200 \mathrm{nmol} / \mathrm{l}$ primer, $200 \mathrm{nmol} / \mathrm{l}$ probe, $200 \mathrm{nmol} / \mathrm{l}$ each of deoxyadenosine triphosphate, deoxycytidine triphosphate, deoxyguanosine triphosphate, deoxythymidine triphosphate, $3.5 \mathrm{mmol} / \mathrm{l} \mathrm{MgCl}_{2}$, and $\times 1$ Taqman Universal PCR Master mix to a final volume of $20 \mu \mathrm{l}$ (all reagents were from PE Applied Biosystems, Foster City, CA). Cycling conditions were $95^{\circ} \mathrm{C}$ for $35 \mathrm{~s}$ and $60^{\circ} \mathrm{C}$ for 30s, followed by 40 cycles at $95^{\circ} \mathrm{C}$ for $15 \mathrm{~s}$ and $60^{\circ} \mathrm{C}$ for $1 \mathrm{~min}$. Relative gene expression levels are expressed as ratios (differences between the $\mathrm{Ct}$ values) between two absolute measurements (PSF3/ $\beta$-actin).

\section{Follow-up}

The follow-up period was defined as the interval between the date of operation and the date of the patient's death or the last visit. The follow-up time ranged from 7 months to 73 months (median, 41 months). All patients were followed until May 2014 with a follow-up rate of $100 \%$. Disease-free survival was measured from the day of surgery to the day of the first evidence of tumor recurrence or metastasis. Overall survival was measured from the day of surgery to the day of death.

\section{Statistical analysis}

Associations between PSF3 expression in tumor tissue and clinicopathological features were determined using the $x^{2}$-test. Survival was examined using the Kaplan-Meier method, and the significance of the difference was evaluated by a log-rank test. A Cox regression analysis was carried out to assess independent prognostic factors for diseasefree survival and overall survival in colorectal cancer. All statistical calculations were performed using SPSS software (SPSS 17.0, Chicago, IL, USA) and $P<0.05$ was considered statistically significant.

\section{Results}

\section{PSF3 expression level in colorectal cancer}

The mRNA expression level of PSF3 were determined in 137 colorectal cancer and the adjacent normal tissues by qRT-PCR. Median mRNA expression levels were $1.35 \times$ $10^{-3}$ (range $2.88 \times 10^{-4}$ to $3.16 \times 10^{-2}$ ) for tumor tissues and $2.94 \times 10^{-4}$ (range $5.48 \times 10^{-5}$ to $1.27 \times 10^{-3}$ ) for adjacent normal tissues, and the differences were statistically significant $(P<0.05)$. To evaluate the role of PSF3 in colorectal cancer, we investigated whether PSF3 expression was associated with any of clinicopathological variables in the 137 enrolled cases of colorectal cancer. By adopting cut-off value according to median PSF3 expression level, we found that PSF3 expression was significantly associated with tumor size, depth of wall invasion, lymph node metastasis, TNM stage, tumor differentiation, and five-year survival. No significant relationship was noted between PSF3 expression and age, gender, distant metastasis, and adjuvant chemotherapy (Table 1).

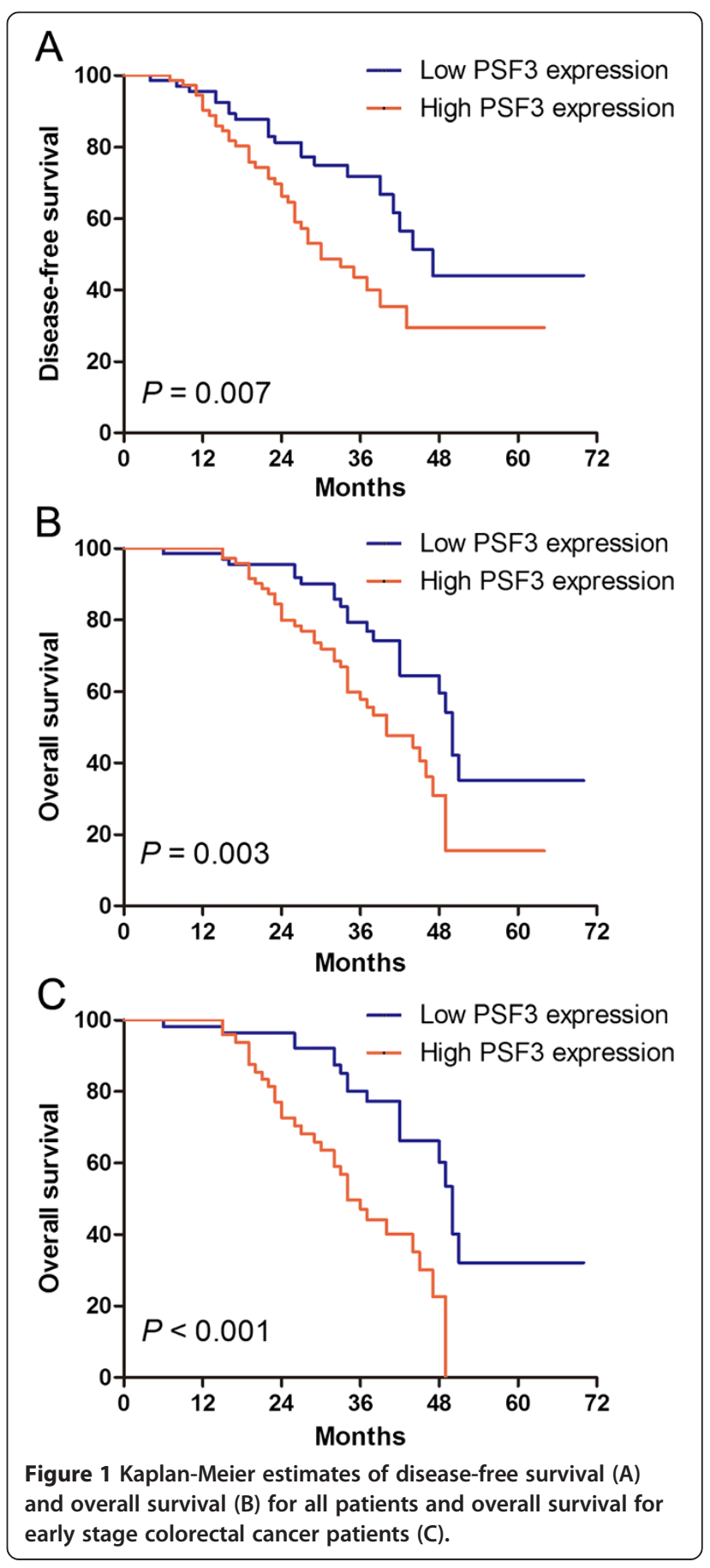


High expression of PSF3 was associated with poor patient prognosis

Using the data collected from 137 patients, we evaluated their prognosis and its relationship to the expression of PSF3. The disease-free survival in patients with low PSF3 levels $(39.5 \pm 7.2$ months $)$ was significantly longer than that in patients with high levels $(28.6 \pm 6.4$ months $)(P=0.007$; Figure 1A). Univariate analysis initially included age, gender, tumor size, depth of wall invasion, lymph node metastasis, distant metastasis, tumor differentiation, TNM stage, adjuvant chemotherapy, and PSF3 expression level for disease-free survival analysis. The tumor size, lymph node metastasis, distant metastasis, TNM stage, tumor differentiation, adjuvant chemotherapy, and PSF3 expression level were associated with disease-free survival and were introduced into the multivariate analysis (Table 2). In the multivariate analysis, late TNM stage, poor differentiation, and high PSF3 level were shown to have a statistically independent prognostic value with respect to disease-free survival (Table 2). In addition, we also examined the overall survival of PSF3 low level and PSF3 high level groups and found a statistically difference between the two groups by using the log-rank test $(P=0.003)$. The median survival time of patients with low PSF3 levels (59.7 \pm 13.8 months) was significantly longer than that of patients with high PSF3 levels (47.2 \pm 11.4 months) (Figure 1B). A univariate analysis indicated that among the clinicopathological factors, tumor size, lymph node metastasis, distant metastasis, tumor differentiation, TNM stage, adjuvant chemotherapy, and PSF3 expression level were correlated with the outcome (Table 3). Further assessment using the Cox multivariate analysis indicated that distant metastasis, poor differentiation, late TNM stage, and high PSF3 expression were statistically significant predictors for poor overall survival (Table 3).
High expression of PSF3 was also associated with poor patient prognosis in early stage colorectal cancer

In the current study, we further analyzed the association of PSF3 expression in early stage (stage I and II) colorectal cancer. Among the early stage cases, 46 and 54 patients were classified as high PSF3 level and low PSF3 level, respectively. A survival analysis that included only early stage patients revealed that the overall survival for the low PSF3 expression group was longer than that for the high PSF3 expression group. The log-rank test showed that the intergroup difference was statistically significant $(P<0.001$; Figure $1 C)$.

\section{Discussion}

PSF3 is a member of the GINS complex, along with Sld5, PSF1, and PSF2. PSF1 is tightly regulated at the transcriptional level in stem cells and enables the successful detection of cancer stem cells [9-12]. Therefore, it seems reasonable that other GINS components may also facilitate the detection of cancer stem cells in tumors. Cancer stem cells, which are resistant to anti-cancer drugs and irradiation, appear to be responsible for tumor growth in hematological and solid cancers. The detection of these cells is critical for identifying molecular targets to inhibit their growth. Previous study has shown that all GINS components are overexpressed in intrahepatic cholangiocarcinoma tissues and PSF3 is also increased in lung adenocarcinoma [17]. To our knowledge, this is the first study to detect PSF3 expression in colorectal cancer and to show that PSF3 expression might be a useful prognostic marker for assessing patient survival in colorectal cancer.

In this study, we performed qRT-PCR of surgically resected colorectal cancer specimens to determine the PSF3 status in cancer tissues clinically. The results revealed that PSF3 expression was higher in the colorectal

Table 2 Univariate and multivariate analyses of the association between the disease-free survival of 137 patients with colorectal cancer

\begin{tabular}{|c|c|c|c|c|}
\hline \multirow[t]{2}{*}{ Variables } & \multicolumn{2}{|c|}{ Univariate analysis } & \multicolumn{2}{|c|}{ Multivariate analysis } \\
\hline & $\mathrm{HR}(95 \% \mathrm{Cl})$ & $P$-value & $\mathrm{HR}(95 \% \mathrm{Cl})$ & $P$-value \\
\hline Age & $1.02(0.94-1.13)$ & 0.748 & & \\
\hline Gender & $0.95(0.87-1.09)$ & 0.396 & & \\
\hline Tumor size & $1.32(1.03-1.58)$ & 0.044 & $1.25(0.98-1.46)$ & 0.067 \\
\hline Depth of wall invasion & $1.14(0.96-1.35)$ & 0.185 & & \\
\hline Lymph node metastasis & $1.74(1.23-2.28)$ & 0.006 & $1.27(0.94-1.54)$ & 0.083 \\
\hline Distant metastasis & $1.56(1.19-1.77)$ & 0.013 & $1.33(0.96-1.67)$ & 0.075 \\
\hline TNM stage & $1.91(1.54-2.60)$ & $<0.001$ & $1.78(1.21-2.13)$ & $0.008^{*}$ \\
\hline Differentiation & $1.66(1.31-2.05)$ & 0.002 & $1.45(1.10-1.78)$ & $0.028^{*}$ \\
\hline Adjuvant chemotherapy & $1.25(1.06-1.47)$ & 0.037 & $1.08(0.87-1.29)$ & 0.154 \\
\hline PSF3 expression & $1.83(1.55-2.17)$ & $<0.001$ & $1.66(1.36-2.02)$ & $0.003^{*}$ \\
\hline
\end{tabular}

Cl confidence interval, HR hazard ratio, PSF3 partner of Sld five 3.

*Significant $P$-value in multivariate analysis. 
Table 3 Univariate and multivariate analyses of the association between the overall survival of 137 patients with colorectal cancer

\begin{tabular}{|c|c|c|c|c|}
\hline \multirow[t]{2}{*}{ Variables } & \multicolumn{2}{|c|}{ Univariate analysis } & \multicolumn{2}{|c|}{ Multivariate analysis } \\
\hline & $\mathrm{HR}(95 \% \mathrm{Cl})$ & $P$-value & $\mathrm{HR}(95 \% \mathrm{Cl})$ & $P$-value \\
\hline Age & $1.12(0.94-1.31)$ & 0.576 & & \\
\hline Gender & $0.99(0.87-1.14)$ & 0.834 & & \\
\hline Tumor size & $1.41(1.13-1.68)$ & 0.033 & $1.04(0.89-1.18)$ & 0.473 \\
\hline Depth of wall invasion & $1.33(0.98-1.70)$ & 0.065 & & \\
\hline Lymph node metastasis & $1.47(1.08-1.85)$ & 0.014 & $1.24(0.96-1.47)$ & 0.085 \\
\hline Distant metastasis & $1.54(1.22-1.92)$ & 0.007 & $1.38(1.12-1.63)$ & $0.006^{*}$ \\
\hline TNM stage & $1.67(1.39-1.94)$ & $<0.001$ & $1.48(1.15-1.77)$ & $0.004^{*}$ \\
\hline Differentiation & $1.41(1.18-1.65)$ & 0.009 & $1.26(1.02-1.51)$ & $0.041^{*}$ \\
\hline Adjuvant chemotherapy & $1.37(1.05-1.74)$ & 0.023 & $1.14(0.91-1.39)$ & 0.093 \\
\hline PSF3 expression & $1.59(1.26-1.95)$ & $<0.001$ & $1.35(1.10-1.64)$ & $0.002^{*}$ \\
\hline
\end{tabular}

Cl confidence interval, HR hazard ratio, PSF3 partner of Sld five 3.

* Significant $P$-value in multivariate analysis.

cancer specimens than in adjacent normal tissues. In order to elucidate the role of high PSF3 expression on the prognosis of patients with colorectal cancer, a prognostic analysis was carried using the patients' follow-up data. Survival analysis revealed that the disease-free survival and overall survival in patients with low PSF3 expression was notably longer than that of patients with high PSF3 expression. These findings indicated that high PSF3 expression significantly affected the clinical course and was correlated with malignant behavior of tumors. The significance of PSF3 expression on these clinical features was also supported by our analysis of the relationship between PSF3 expression and clinicopathological characteristics of 137 patients. Cox multivariate analysis indicated that high PSF3 expression was the most significant predictor of poor prognosis, rather than the TNM stage or tumor differentiation. Furthermore, a prognostic analysis that included only early stage cases (stage I and II) revealed that the overall survival rate of the high PSF3 expression group was significantly lower than that of the low PSF3 expression group. These findings suggest that high PSF3 expression may be used as a reference index for molecular staging of patients with a high risk of death and thereby likely to benefit from intensive adjuvant therapy.

What is the basis of the relationship between high PSF3 expression and poor prognosis? We believe that high PSF3 expression may be related to cancer cell proliferation because PSF3 was required in the early stage of DNA replication, along with other GINS members [9-12]. A previous study by Nagahama et al. found high expression of PSF3 in several colon carcinoma cell lines (HCT116, colo320DM, SW837, and HT-29) and that PSF3 gene knock-down in these cell lines resulted in growth inhibition characterized by delayed S-phase progression [16].
The results suggested that PSF3 marks malignant colon cancer and has a role in cancer cell proliferation.

\section{Conclusions}

In conclusion, we have shown that high PSF3 expression plays an important role in the progression of colorectal cancer and acts as a factor significantly affecting the prognosis of patients. These results suggested that PSF3 could be used as a reference index for the molecular staging to select patients at high risk of death and relapsed patients who may benefit from intensive adjuvant therapy.

\section{Abbreviations}

PSF3: Partner of SId five 3; GINS: Go-Ichi-Ni-San; mRNA: Messenger RNA; MCM: Minichromosome maintenance; qRT-PCR: Quantitative reversetranscription polymerase chain reaction; cDNA: Complementary DNA.

Competing interests

The authors declare that they have no competing interests.

\section{Authors' contributions}

XS and WS carried out the qRT-PCR experiments and drafted the manuscript. $\mathrm{MH}$ and $\mathrm{YW}$ collected the clinical data. YX participated in the design of the study and performed the statistical analysis. ZW conceived of the study, and participated in its design and coordination and helped to draft the manuscript. All authors read and approved the final manuscript.

\section{Acknowledgments}

This work was supported by a grant from Yantai Postdoctoral Science Foundation (No. 57632411).

\section{Author details}

${ }^{1}$ Department of Laboratory, Yuhuangding Hospital, No. 20 Yuhuangding East Road, Yantai 264000, Shandong, China. ${ }^{2}$ General Surgery, Yuhuangding Hospital, No. 20 Yuhuangding East Road, Yantai 264000, Shandong, China. ${ }^{3}$ Anorectal Surgery, Yuhuangding Hospital, No. 20 Yuhuangding East Road, Yantai 264000, Shandong, China.

Received: 26 September 2014 Accepted: 26 October 2014

Published online: 18 November 2014 
1. Takayama Y, Kamimura Y, Okawa M, Muramatsu S, Sugino A, Araki H: GINS a novel multiprotein complex required for chromosomal DNA replication in budding yeast. Genes Dev 2003, 17:1153-1165.

2. Moyer SE, Lewis PW, Botchan MR: Isolation of the Cdc45/Mcm2-7/GINS (CMG) complex, a candidate for the eukaryotic DNA replication fork helicase. Proc Natl Acad Sci U S A 2006, 103:10236-10241.

3. Pacek M, Tutter AV, Kubota Y, Takisawa H, Walter JC: Localization of MCM2-7, Cdc45, and GINS to the site of DNA unwinding during eukaryotic DNA replication. Mol Cell 2006, 21:581-587.

4. Chang YP, Wang G, Bermudez V, Hurwitz J, Chen XS: Crystal structure of the GINS complex and functional insights into its role in DNA replication. Proc Natl Acad Sci U S A 2007, 104:12685-12690.

5. Kamada K, Kubota Y, Arata T, Shindo Y, Hanaoka F: Structure of the human GINS complex and its assembly and functional interface in replication initiation. Nat Struct Mol Biol 2007, 14:388-396.

6. Kubota Y, Takase Y, Komori Y, Hashimoto Y, Arata T, Kamimura Y, Araki H, Takisawa H: A novel ring-like complex of Xenopus proteins essential for the initiation of DNA replication. Genes Dev 2003, 17:1141-1152.

7. Barkley LR, Song IY, Zou Y, Vaziri C: Reduced expression of GINS complex members induces hallmarks of pre-malignancy in primary untransformed human cells. Cell Cycle 2009, 8:1577-1588

8. Hemerly AS, Prasanth SG, Siddiqui K, Stillman B: Orc1 controls centriole and centrosome copy number in human cells. Science 2009, 323:789-793.

9. Ueno $M$, Itoh M, Kong L, Sugihara K, Asano M, Takakura N: PSF1 is essential for early embryogenesis in mice. Mol Cell Biol 2005, 25:10528-10532.

10. Ueno M, Itoh M, Sugihara K, Asano M, Takakura N: Both alleles of PSF1 are required for maintenance of pool size of immature hematopoietic cells and acute bone marrow regeneration. Blood 2009, 113:555-562.

11. Han $Y$, Ueno M, Nagahama $Y$, Takakura N: Identification and characterization of stem cell-specific transcription of PSF1 in spermatogenesis. Biochem Biophys Res Commun 2009, 380:609-613.

12. Nagahama $Y$, Ueno M, Miyamoto S, Morii E, Minami T, Mochizuki N, Saya $H_{1}$ Takakura N: PSF1, a DNA replication factor expressed widely in stem and progenitor cells, drives tumorigenic and metastatic properties. Cancer Res 2010, 70:1215-1224

13. Obama K, Ura K, Satoh S, Nakamura Y, Furukawa Y: Up-regulation of PSF2, a member of the GINS multiprotein complex, in intrahepatic cholangiocarcinoma. Oncol Rep 2005, 14:701-706.

14. Nakahara I, Miyamoto M, Shibata T, Akashi-Tanaka S, Kinoshita T, Mogushi K, Oda K, Ueno M, Takakura N, Mizushima H, Tanaka H, Ohta T: Up-regulation of PSF1 promotes the growth of breast cancer cells. Genes Cells 2010, 15:1015-1024.

15. Ryu B, Kim DS, Deluca AM, Alani RM: Comprehensive expression profiling of tumor cell lines identifies molecular signatures of melanoma progression. PLoS One 2007, 2:e594.

16. Nagahama $Y$, Ueno M, Haraguchi N, Mori M, Takakura N: PSF3 marks malignant colon cancer and has a role in cancer cell proliferation. Biochem Biophys Res Commun 2010, 392:150-154.

17. Hokka D, Maniwa Y, Tane S, Nishio W, Yoshimura M, Okita Y, Ohbayashi C, Sakai Y, Chen X, Hayashi Y: Psf3 is a prognostic biomarker in lung adenocarcinoma. Lung Cancer 2013, 79:77-82.

doi:10.1186/s13000-014-0217-5

Cite this article as: Sun et al:: Partner of Sld five 3: a potential prognostic biomarker for colorectal cancer. Diagnostic Pathology 2014 9:217.

\section{Submit your next manuscript to BioMed Central and take full advantage of:}

- Convenient online submission

- Thorough peer review

- No space constraints or color figure charges

- Immediate publication on acceptance

- Inclusion in PubMed, CAS, Scopus and Google Scholar

- Research which is freely available for redistribution 